\title{
Desynchronization Of Stochastically Synchronized Chemical Oscillators
}

\author{
Razan Snari
}

Mark R. Tinsley

Dan Wilson

Sadegh Faramarzi

Theoden Ivan Netoff

See next page for additional authors

Follow this and additional works at: https://researchrepository.wvu.edu/faculty_publications

\section{Digital Commons Citation}

Snari, Razan; Tinsley, Mark R.; Wilson, Dan; Faramarzi, Sadegh; Netoff, Theoden Ivan; Moehlis, Jeff; and Showalter, Kenneth, "Desynchronization Of Stochastically Synchronized Chemical Oscillators" (2015). Faculty Scholarship. 499.

https://researchrepository.wvu.edu/faculty_publications/499 
Authors

Razan Snari, Mark R. Tinsley, Dan Wilson, Sadegh Faramarzi, Theoden Ivan Netoff, Jeff Moehlis, and Kenneth Showalter 


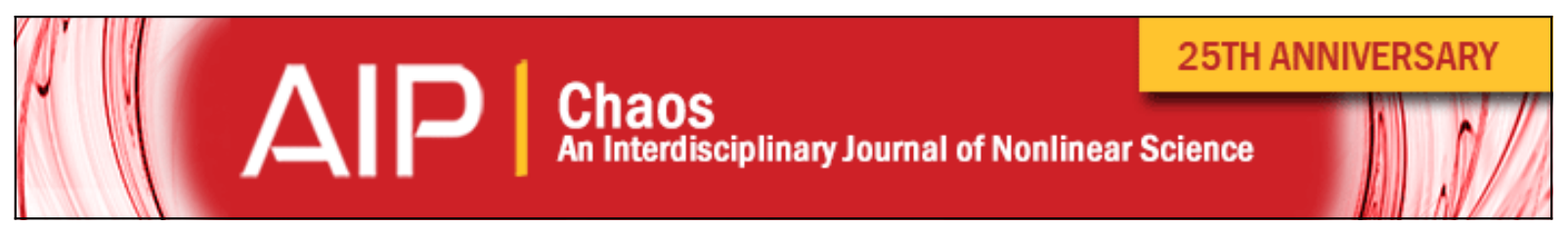

\section{Desynchronization of stochastically synchronized chemical oscillators}

Razan Snari, Mark R. Tinsley, Dan Wilson, Sadegh Faramarzi, Theoden Ivan Netoff, Jeff Moehlis, and Kenneth

Showalter

Citation: Chaos 25, 123116 (2015); doi: 10.1063/1.4937724

View online: http://dx.doi.org/10.1063/1.4937724

View Table of Contents: http://scitation.aip.org/content/aip/journal/chaos/25/12?ver=pdfcov

Published by the AIP Publishing

\section{Articles you may be interested in}

Thermally induced synchronization and stochastic resonance between magnetization regimes in spin-transfer nano-oscillators

J. Appl. Phys. 111, 07 C915 (2012); 10.1063/1.3679008

Spatiotemporal Organization of Stochastic Synchronization

AIP Conf. Proc. 665, 200 (2003); 10.1063/1.1584892

Stochastic phase resetting of stimulus-locked responses of two coupled oscillators: Transient response clustering, synchronization, and desynchronization

Chaos 13, 364 (2003); 10.1063/1.1505813

Frequency and phase synchronization in stochastic systems

Chaos 13, 225 (2003); 10.1063/1.1500497

Synchronization in ensembles of stochastic resonators

AIP Conf. Proc. 411, 151 (1997); 10.1063/1.54184

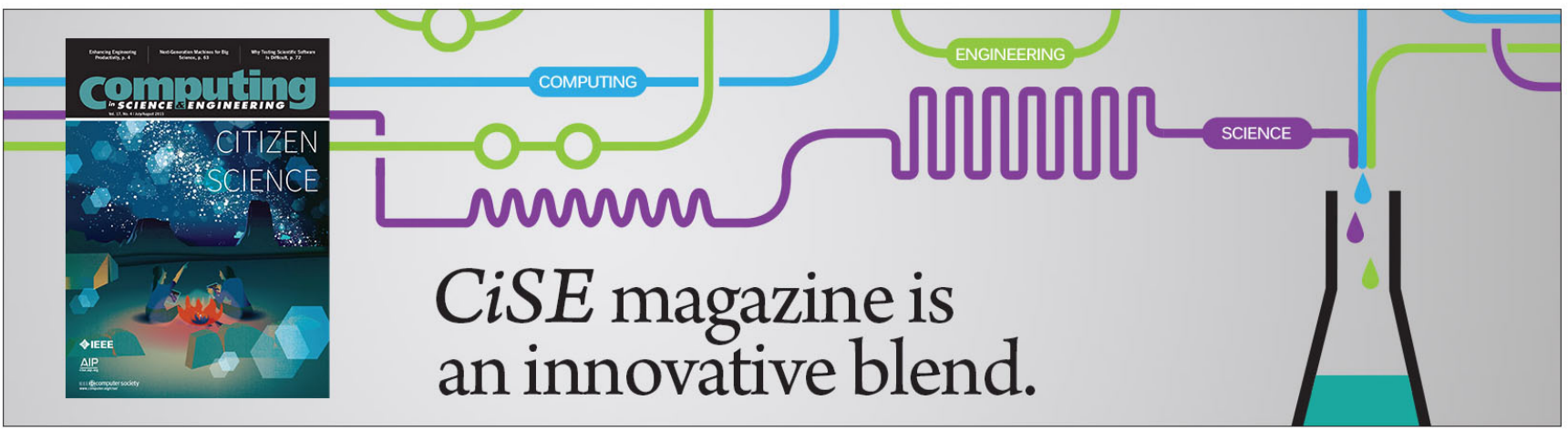




\title{
Desynchronization of stochastically synchronized chemical oscillators
}

\author{
Razan Snari, ${ }^{1}$ Mark R. Tinsley, ${ }^{1, a)}$ Dan Wilson, ${ }^{2}$ Sadegh Faramarzi, ${ }^{1}$ Theoden Ivan Netoff, ${ }^{3}$ \\ Jeff Moehlis, ${ }^{2}$ and Kenneth Showalter ${ }^{1, a)}$ \\ ${ }^{1}$ C. Eugene Bennett Department of Chemistry, West Virginia University, Morgantown, West Virginia \\ 26506-6045, USA \\ ${ }^{2}$ Department of Mechanical Engineering, University of California, Santa Barbara, California 93106, USA \\ ${ }^{3}$ Department of Biomedical Engineering, University of Minnesota, Minneapolis, Minnesota 55455, USA
}

(Received 1 October 2015; accepted 18 November 2015; published online 21 December 2015)

\begin{abstract}
Experimental and theoretical studies are presented on the design of perturbations that enhance desynchronization in populations of oscillators that are synchronized by periodic entrainment. A phase reduction approach is used to determine optimal perturbation timing based upon experimentally measured phase response curves. The effectiveness of the perturbation waveforms is tested experimentally in populations of periodically and stochastically synchronized chemical oscillators. The relevance of the approach to therapeutic methods for disrupting phase coherence in groups of stochastically synchronized neuronal oscillators is discussed. (C) 2015 AIP Publishing LLC. [http://dx.doi.org/10.1063/1.4937724]
\end{abstract}

\begin{abstract}
Stochastic synchronization involves the statistical alignment of the phases of oscillators due to the presence of a common noise signal. It has been proposed as a possible mechanism for pathological phase coherence in communities of neurons. Here, we demonstrate stochastic synchronization in an experimental system of BelousovZhabotinsky chemical micro-oscillators subject to common colored noise. A phase reduction approach, based upon experimentally measured phase response curves, is then used to investigate the design of a suitable signal for effective desynchronization. The designed signal is implemented and tested in the experimental system. Such an approach may lead to improved design and optimization of perturbing stimuli associated with therapeutic methods such as deep brain stimulation.
\end{abstract}

\section{INTRODUCTION}

Synchronous oscillations are important for many physiological processes, ${ }^{1}$ such as hormones synchronized to the diurnal cycle ${ }^{2}$ or communication between populations of neurons during perception navigation. ${ }^{3}$ Many diseases can be linked to dysregulation of synchronous oscillations such as depression, ${ }^{4}$ Parkinson's disease, ${ }^{5}$ and essential tremor. ${ }^{6}$ Therapeutic interventions that help reestablish normal oscillations include timed drug delivery for depression ${ }^{7}$ or electrical stimulation of the brain for Parkinson's disease ${ }^{8}$ and essential tremor. ${ }^{9}$

In the nervous system, voltage oscillations seen at the scalp are thought to emerge from the weak synchronization of large populations of neurons. ${ }^{10,11}$ Synchronization of dynamical oscillators, such as neurons, is typically thought to occur through either mutual coupling or entrainment to a regular common signal. ${ }^{12}$ An alternative form of synchronization that leads to phase coherence is termed stochastic

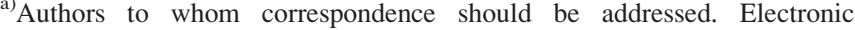
addresses: mark.tinsley@mail.wvu.edu and kshowalt@wvu.edu.
}

synchrony. ${ }^{13-15}$ This type of synchronization, also known as noise-induced synchronization, arises when a set of uncoupled oscillators synchronizes to a common noise input. ${ }^{16}$ Theoretical studies have demonstrated it to be a general behavior, commonly occurring in oscillators with a resetting threshold, which have a propensity to align phases when subject to a common signal. ${ }^{17,18}$ Noise-induced synchronization has been shown to occur for both white noise and other types of non-Gaussian noise that are common in real-world systems. ${ }^{13,14,19-21}$ However, the phenomenon has been subject to only limited experimental investigation. Evidence of noise induced synchronization has been found in vitro in the olfactory bulb, where neurons subject to an aperiodic correlated signal synchronize, ${ }^{22}$ and in bursting of sensory neuronal oscillators of the paddlefish when stimulated with common electrical noise. ${ }^{23}$

Perturbations, using an appropriately designed external driving signal, can desynchronize a system of synchronized oscillators. $^{24-26}$ Several approaches have been proposed to effect such desynchronization. ${ }^{27-29}$ These include the application of a single pulse during the vulnerable phase of the coordinated cycle, double pulse perturbations, and time delayed feedback. ${ }^{18,26,27,30}$ These and other methods have been demonstrated using systems of coupled electrochemical oscillators as well as in numerical simulations of model neurons. ${ }^{18,26,27,30,31}$

It has been hypothesized that the therapeutic mechanism of deep brain stimulation (DBS), used during the treatment of Parkinson's disease, involves the desynchronization of neurons arising from the external periodic electrical stimulation. ${ }^{32}$ DBS has been found to be most effective when its frequency is higher than that of the underlying neuronal oscillators. ${ }^{27}$ An understanding of the mechanism by which it disrupts coordinated neurons is still under debate; ${ }^{33}$ however, computational models suggest that DBS applied at certain frequencies can induce chaotic desynchronization of the neural oscillators. ${ }^{32}$ Assuming a chaotic desynchronization mechanism of therapeutic DBS, it is possible to optimize the 
stimulus patterns by designing waveforms specifically for this purpose. This may improve electrode and battery life by reducing the energy delivered, while potentially improving efficacy and minimizing side effects.

In this work, we will test stimulus waveforms designed to desynchronize oscillators that are synchronized through noise-induced synchronization. We will demonstrate how stimulation delivered at particular phases of an oscillation can advance or retard the oscillation phase, and how the most effective phase for maximizing or minimizing stimulation can be predicted from the phase response curve. This paper will focus on applying stimulation to modulate synchrony in chemical oscillators. We rely upon the direct measurement of the phase response curves (PRCs) to a stimulus input and use the resulting PRCs to design the stimulus waveform to achieve synchronization or desynchronization.

We show both experimentally and in a model system that an appropriately timed low-frequency periodic signal desynchronizes the stochastically synchronized chemical system. The study is conducted in two stages. In order to investigate the importance of the timing of the perturbing signal, a desynchronizing signal is applied at controlled phases to a population of initially synchronized oscillators. The initial synchronization is achieved through entrainment to an appropriate common periodic signal. We then use a common stochastic signal to statistically synchronize a population of uncoupled chemical oscillators and examine the impact of the desynchronizing signal.

\section{EXPERIMENTAL METHODS}

Our experimental system consists of a population of addressable photosensitive Belousov-Zhabotinsky (BZ) chemical micro-oscillators. ${ }^{34}$ The $\mathrm{BZ}$ reaction was first discovered in the 1950s and has since been used extensively as a model system for the investigation of the dynamical properties of many different natural systems. ${ }^{35-38}$ The reaction involves the oxidation of an organic substrate in the presence of a metal catalyst and, under appropriate conditions, exhibits periodic relaxation oscillations. During one cycle of an oscillation, the catalyst changes between oxidized and reduced states. Each of the states has a different color, which allows for convenient monitoring of both the period and phase of an oscillation. In this study, individual BZ microoscillators are created by chemically attaching the metal catalyst to small cation-exchange particles (average radius $=100 \mu \mathrm{m}){ }^{34,39}$ When these particles are placed into a catalyst-free solution, and spatially separated, each particle acts as an independent chemical micro-oscillator. The frequency distribution of the oscillators is predominantly controlled by the underlying size distribution of the cationexchange particles. The gray level $I_{j}$ of each oscillator is monitored with a CCD camera. The use of the photosensitive $\mathrm{Ru}$ (bipy) ${ }_{3}^{2+}$ catalyst allows the manipulation of the phase of the individual oscillators by illumination with a spatial light modulator (SLM). ${ }^{40-42}$

The experimental arrangement is shown in Figure 1. The beam splitter allows the micro-oscillators to be viewed from above using the illumination provided by the
SLM. During an experiment, the light intensity is updated and images are taken every $3 \mathrm{~s}$. Approximately 35 microoscillators are used in each experiment. The oscillators are positioned at least 3 diameters apart in the catalystfree solution to eliminate diffusive coupling. Due to natural aging of the catalyst-free solution, the natural frequency of the oscillators increases during the experiment. The local mean natural frequency of the oscillators can be estimated based upon a subpopulation of five oscillators. These control oscillators are selected at random at the start of an experiment and are subject to a constant light intensity for the duration of the experiment. Their average frequency is calculated throughout the experiment and used as an estimate of the mean natural frequency of the other oscillators.

\section{THEORETICAL BACKGROUND}

The prediction of synchronization or desynchronization by external perturbations is based on the change in the phase difference between two oscillators following a perturbation. The behavior of two oscillators, which are initially entrained to a synchronizing stimulus and then subject to a desynchronizing signal, is analyzed in the Appendix. Assuming that the slower oscillator remains entrained and the faster oscillator escapes entrainment, the phase difference, $\psi$, grows according to

$$
\begin{gathered}
\psi(t) \approx \frac{\Delta \omega+\Omega}{\Lambda}\left(e^{\Lambda t}-1\right), \\
\Lambda=\frac{1}{T} \int_{0}^{T} Z_{-}^{\prime}(\phi(t)) u_{-}(t) d t \\
\Omega=\frac{1}{T} \int_{0}^{T} Z_{-}(\phi(t)) u_{-}(t) d t
\end{gathered}
$$

where $Z_{-}(\phi)$ is the PRC to a $T$-periodic negative external perturbation $u_{-}(t), \Delta \omega=\omega_{0}-2 \pi / T$, where $\omega_{0}$ is the natural frequency of the faster oscillator, $\phi$ is the phase of the

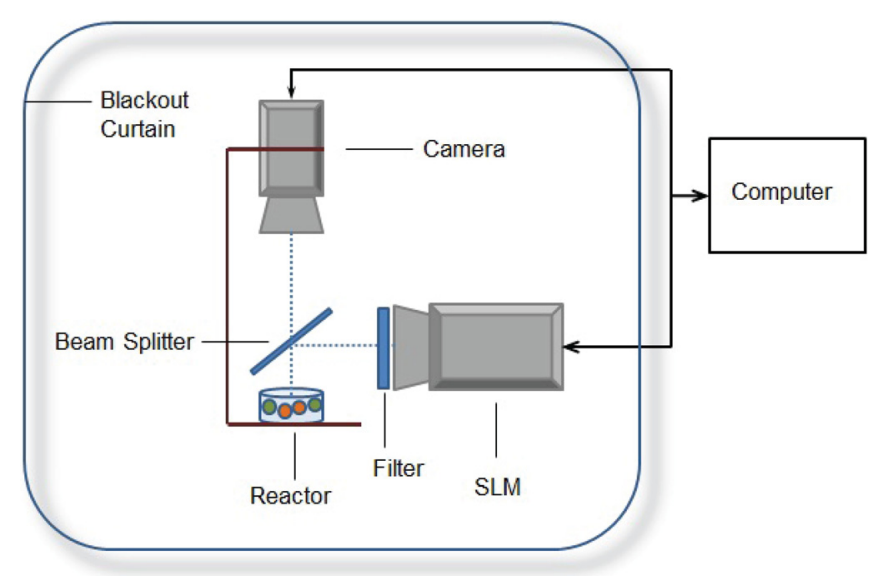

FIG. 1. Experimental Belousov-Zhabotinsky (BZ) chemical system. The photosensitive micro-oscillators are monitored with the CCD camera and are illuminated with the SLM according to an illumination algorithm. 
slower oscillator, and ' $\equiv d / d \phi$. The finite time Lyapunov exponent, $\Lambda$, determines the rate of exponential desynchronization between the two oscillators (cf. Refs. 28 and 43).

Figure 2(a) shows an experimentally obtained PRC, in response to a negative perturbation, using the "direct method. ${ }^{, 44,45}$ The PRC is constructed by applying an intermittent perturbation, of duration $\Delta t=2 \mathrm{~s}$, to a set of independent micro-oscillators, allowing at least one unperturbed period between perturbations. The phase of each oscillator at the time of perturbation is determined by assuming the period is the same as that of the previous cycle, during which no perturbation occurred. The unperturbed period also allows the phase advance/retardation $\Delta \phi$ to be calculated. The points in the PRC are then calculated according to $Z(\phi)=\frac{\Delta \phi}{u \Delta t}$, where $u$ is the absolute value of the amplitude and $\Delta t$ is the duration of the perturbation used to construct the PRC. In the case of Figure 2(a), the background illumination applied to the oscillators is 180 gray scale units; during a perturbation, the light intensity is reduced to 0 gray scale units. The data points in the interval $\phi=[0,4.4]$ follow a linear trend and a line of best fit, shown in green, is obtained using linear regression.

Owing to the positive slope of the PRC, any signal applied during this initial portion of an oscillatory cycle will lead to phase separation of a pair of synchronized oscillators. The values of $\Lambda$ and $\Omega$ associated with such a signal can be approximated using $\phi(t)=\omega t$, where $\omega=2 \pi / T$, which is valid in the limit that the magnitude of the external stimulus is small (a similar approximation was used in Refs. 28 and 29), in conjunction with Eq. (1) and the linear fit shown in Figure 2(a).

In contrast, application of a negative stimulus towards the end of the oscillatory cycle will lead to a negative value of $\Lambda$. Similar "resetting" behavior is found if the PRC associated with a positive perturbation is used, Figure 2(b). In the

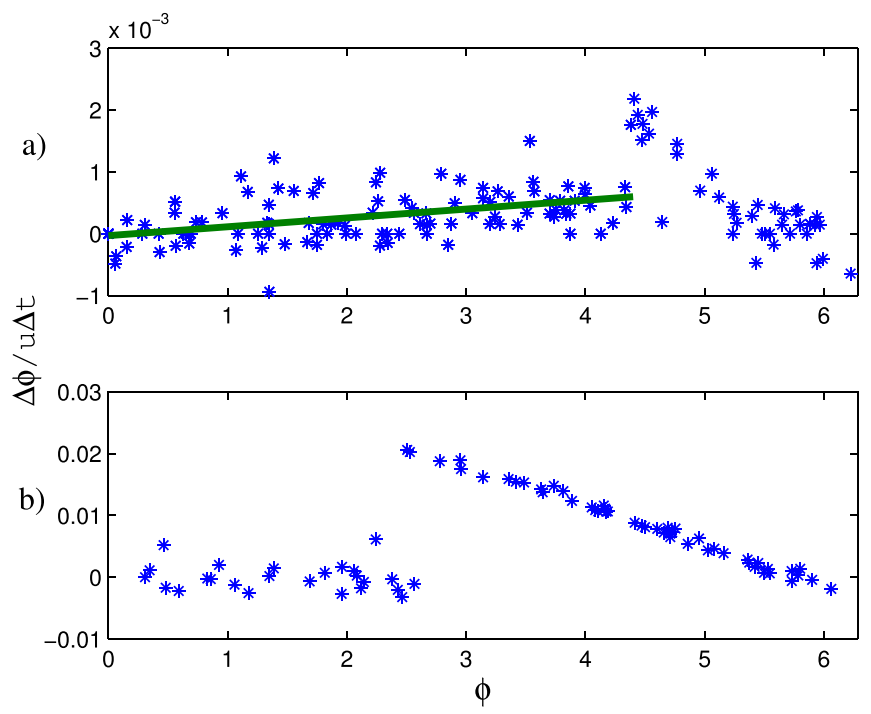

FIG. 2. Phase response curves (PRCs) showing change in phase as a function of phase at which a perturbation is applied. (a) The PRC corresponding to negative perturbations. A line, $Z \times 10^{4}=1.50 \phi-0.04$, is fitted to the data in the region $[0,4.4]$. (b) The positive perturbation PRC. The negative and positive perturbations have amplitudes of 180 and 70 gray scale units $\left(1.67 \mathrm{~mW} \mathrm{~cm}^{-2}\right.$ and $\left.0.64 \mathrm{~mW} \mathrm{~cm}^{-2}\right)$, respectively, and duration of $2 \mathrm{~s}$. The baseline intensity is 180 gray scale units. The composition of the catalystfree solution is $\left[\mathrm{NaBrO}_{3}\right]=0.48 \mathrm{M},[\mathrm{MA}]=0.08 \mathrm{M},[\mathrm{NaBr}]=0.02 \mathrm{M}$, and $\left[\mathrm{H}_{2} \mathrm{SO}_{4}\right]=0.78 \mathrm{M}$. interval $[0,2.7], \Delta \phi$ of this positive perturbation PRC is approximately zero, while during the remainder of the cycle the PRC has a constant negative slope. Thus, both a positive perturbation and a late, in the phase cycle, negative perturbation will enhance synchronization in this chemical system.

\section{EXPERIMENTAL RESULTS: PERIODIC ENTRAINMENT SIGNAL}

The micro-oscillators are initially synchronized by entrainment to a common periodic positive pulse light signal. Figure 3(a) shows the projected light intensity that is applied as a repeated positive pulse above the background light intensity. The periodic positive pulse is introduced at $\mathrm{t}=120 \mathrm{~s}$. Each positive pulse is of a fixed amplitude and lasts for $9.0 \mathrm{~s}$, after which the background intensity is restored. The frequency of the forcing signal was chosen to be slightly higher than the mean frequency of the control oscillators because our preliminary studies indicated that this leads to the most effective entrainment. In order to accommodate drift in the natural frequency of the oscillators during an experiment, the frequency of the common light signal is continuously updated based upon the natural frequency of the control oscillators.

The oscillators rapidly entrain to this signal and synchronize with each other. This is indicated by the increase in the amplitude of the mean signal, shown in Figure 3(a), as well as the alignment of the individual oscillator signals and the increase in the order parameter, Figures 3(b) and 3(c). The peak in the mean oscillator signal typically occurs between 6 and $9 \mathrm{~s}$ after the start of each light pulse. At $t=1200 \mathrm{~s}$, a periodic negative pulse train is added to the common signal in an attempt to desynchronize the system. This negative pulse train is phase locked relative to the positive perturbations. The chemistry of the system is less sensitive to a reduction in light intensity, and we, therefore, make the negative pulse longer in duration. The PRC analysis outlined above suggests that negative pulses should be applied in the first half of the phase cycle of the synchronized oscillators to lead to effective desynchronization. The applied signal is shown in Figure 3(a), which is phase locked to the entraining signal so that it occurs during the first half of the entraining signal cycle. The negative pulses quickly lead to phase advancement of a number of the oscillators, breaking the synchrony of the original group of oscillators. This can be seen in the individual time series plots, Figure 3(b), as well as by the reduction in the peak amplitude of the mean signal, Figure 3(a). Figure 3(c) shows the corresponding changes in the Kuramoto order parameter, with a large decrease in its value after $\mathrm{t}=1200 \mathrm{~s}$ from close to 1.0 to 0.46 .

The importance of the timing of the applied negative perturbations can be further demonstrated. According to the PRC analysis, if the timing of the applied perturbation corresponds to a region where the PRC has a negative slope (negative Lyapunov exponent), then the perturbation will support synchronization, rather than leading to desynchronization. This is confirmed in Figure 3(d), which shows the result of applying negative pulses that are timed to occur towards the end of the entraining signal cycle. The mean signal and individual time series of ten representative oscillators show that 

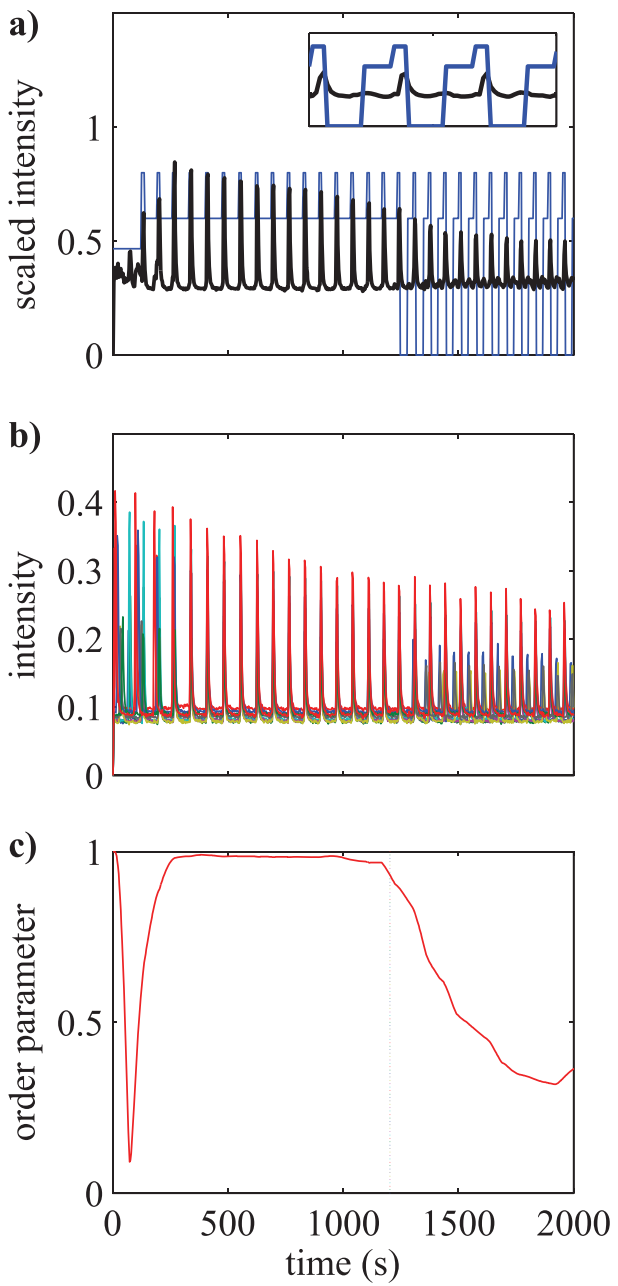

e)

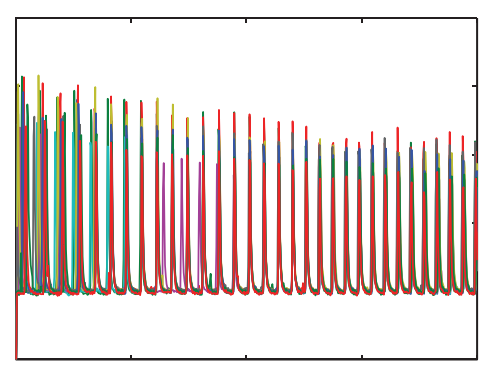

f)

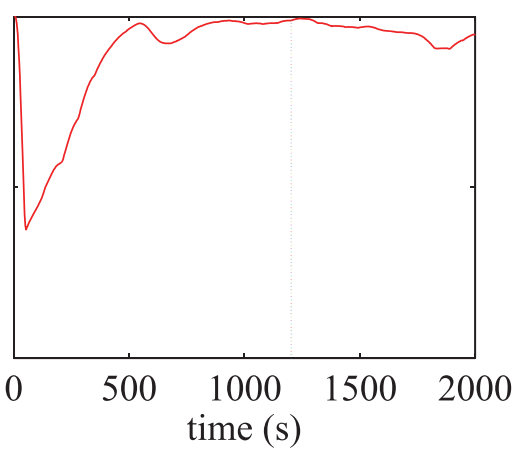

FIG. 3. Effect of periodic perturbations on oscillators entrained by periodic signal. (a) A common periodic pulse light signal (blue line), of duration $9.0 \mathrm{~s}$, is initiated at $\mathrm{t}=120 \mathrm{~s}$. A second perturbation, in which the light intensity is decreased for a duration of $1 / 2$ of the synchronizing signal period, is introduced at $\mathrm{t}=1200 \mathrm{~s}$. It is timed to occur in the first half of the entraining signal cycle. Mean oscillator signal (black line). (b) Individual time series of ten representative oscillators. (c) Kuramoto order parameter. The forcing signal has a period that is $9.0 \mathrm{~s}$ shorter than the mean natural period of the control oscillators. (d)-(f) The same as (a)-(c) except the dark signal is timed to occur in the latter half of the synchronizing cycle. The mean natural frequency of the control oscillators changes during the experiment from $81 \mathrm{~s}$ to $72 \mathrm{~s}$ (a)-(c) and $75 \mathrm{~s}$ to $65 \mathrm{~s}$ (d)-(f). The experiments use 30 micro-oscillators and 5 control micro-oscillators. The baseline, positive pulse, and negative pulse light intensities are 180,240 , and 0 gray scale units $\left(1.67,2.23\right.$, and $0 \mathrm{~mW} \mathrm{~cm} \mathrm{~cm}^{-2}$ ), respectively. Chemical concentrations as in Figure 2. Inset in (a) and (d) shows blowup of the periodic perturbations and oscillator response for $1500 \mathrm{~s}-1700 \mathrm{~s}$. a large amplitude regular mean signal and coherent firing are maintained. The order parameter during the experiment remains close to 1.0 , indicating sustained synchronization after applying negative pulses in the latter half of the phase cycle.

\section{EXPERIMENTAL RESULTS: NOISE ENTRAINMENT SIGNAL}

The behavior of the oscillators in the absence of any imposed signal is shown in Figure 4. The mean signal of the oscillators is aperiodic with a small amplitude, Figure 4(a). The individual oscillators proceed at their natural frequencies, leading to a low value of the order parameter, Figures 4(b) and 4(c). In Sec. IV, we saw that such oscillators are entrained to a common periodic signal. In this section, the response of the oscillators to a common noise signal is explored. An exponentially correlated noise signal $X(t)$, with mean value $X_{0}$, can be generated using the OrnsteinOhlenbeck equation ${ }^{14,46}$

$$
\dot{X}(t)=-\theta\left(X(t)-X_{0}\right)+\sqrt{2 \theta \sigma^{2}} \eta(t),
$$

where $\theta$ determines the rate at which the process reverts to the mean, and $\sigma^{2}$ determines its variance. The i.i.d. (independent and identically distributed) Gaussian white noise, $\eta$, has a zero mean and autocorrelation $\langle X(t) X(s)\rangle=\delta(t-s)$.
A typical noise signal produced using the Euler-Maruyama $\operatorname{method}^{47}$ and a random initial value $X(0)$ is shown in Figure 4(d). Figures 4(e) and 4(f) show the behavior of the individual oscillators in the presence of this common noise signal. Regions of strong intermittent phase coherence among the individual oscillator signals are seen together with corresponding increases in the mean signal amplitude and order parameter. Comparison of the time average order parameter in Figure 4(c), with no common noise present, to the time average order parameter in Figure 4(f), with common noise present, reveals an increase from 0.15 to 0.57 . The behavior is better characterized statistically by finding the mean of the individual time averaged order parameters obtained from separate experiments. Each experiment uses a different set of micro-oscillators and a different random initial condition for the noise signal. Based upon this approach, the mean time averaged order parameter was found to be $0.16 \pm 0.03$, with no common noise present, compared to $0.53 \pm 0.08$, with common noise present. The statistics are based upon ten separate experiments of each type. Statistical comparison (t-test) of these mean order parameter values shows them to be significantly different, providing strong evidence of noise induced synchronization in the system.

The impact of a periodic negative pulse signal on the noise induced synchronized oscillators is shown in Figure 5. The frequency of the signal is again determined based upon the natural frequency of five control oscillators. The timing of the pulse relative to the phase of the oscillators now 

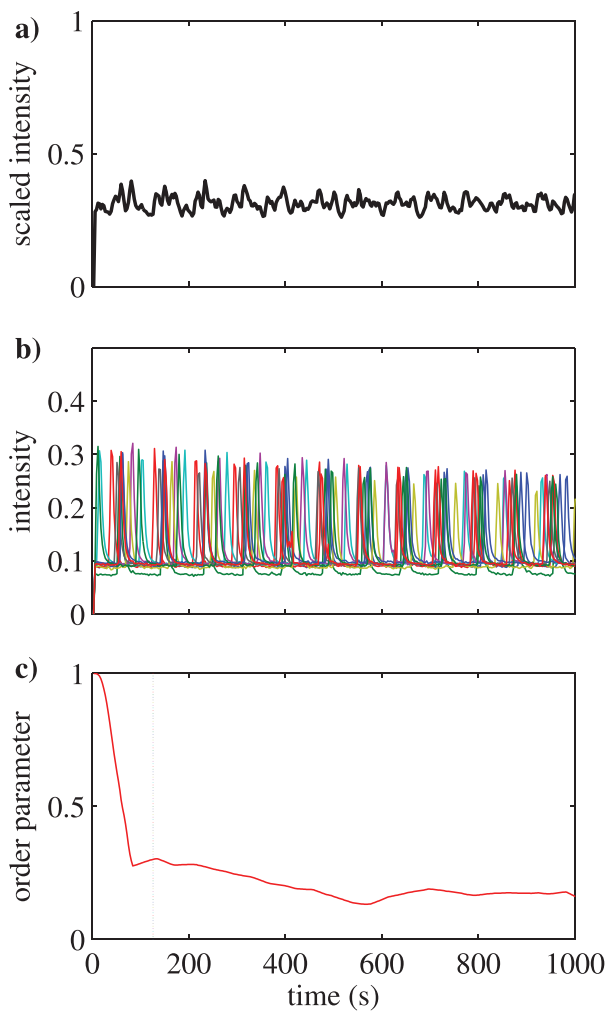

f)

e)
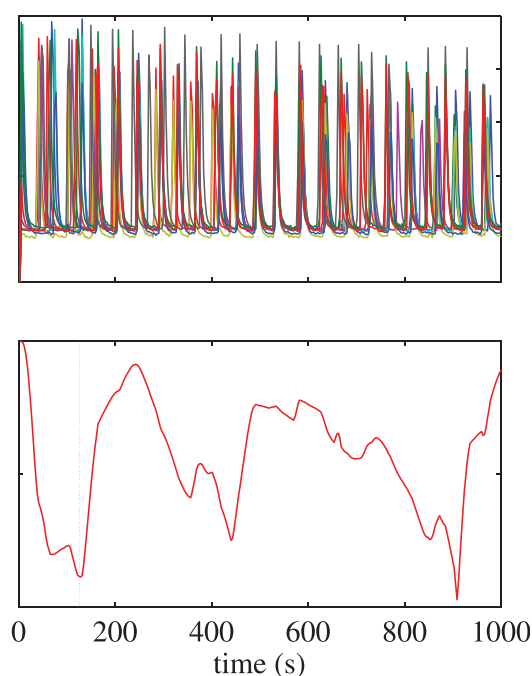

FIG. 4. Oscillations without any entrainment signal and in the presence of common colored noise signal. (a) Mean signal of 30 oscillators, black line. (b) Individual time traces of 10 representative oscillators. (c) Kuramoto order parameter. (d) Mean signal of 30 oscillators, black line, in the presence of a common colored noise signal. The noise signal scaled between 0 and 1 , blue line. (e) and (f) Same as (b) and (c) except in the presence of the noise signal shown in (d). Each experiment has a total duration of $2000 \mathrm{~s}$, though for clarity only $1000 \mathrm{~s}$ are shown. Noise parameters: $\theta=0.08, \sigma=50$, background light intensity $=140$ gray scale units $\left(1.29 \mathrm{~mW} \mathrm{~cm}^{-2}\right)$. cannot be predetermined. It is clear from Figure 5 that the application of the negative pulses leads to a decrease in average amplitude of the mean signal and a decrease in the timeaveraged order parameter. As previously, the experiment is repeated ten times and the mean time-averaged order parameter is determined to be $0.27 \pm 0.05$. Statistical comparison (t-test) of this value against either the noise-free value or the common noise value indicates it to be significantly different from both of them. This indicates that the application of the negative pulses leads to a degree of desynchronization, though not complete incoherence as seen in the system with no common noise.

The important role associated with the slope of the PRC is demonstrated by examining the impact of a positive pulse train, as shown in Figures 5(d)-5(f). The addition of positive pulses on top of the noise signal leads to effective synchronization of the oscillators. Following an initial transient, the order parameter has a value close to 1.0 for the remainder of the experiment.

If the desynchronizing signal is applied repeatedly, the oscillators will eventually move to a cluster state, as in a)

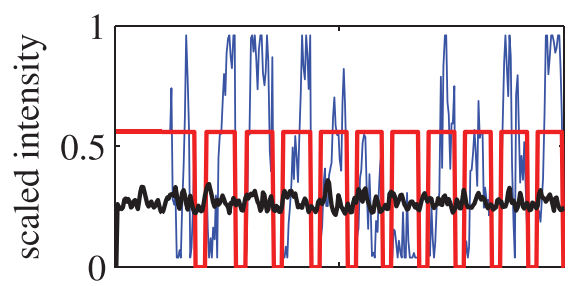

b)

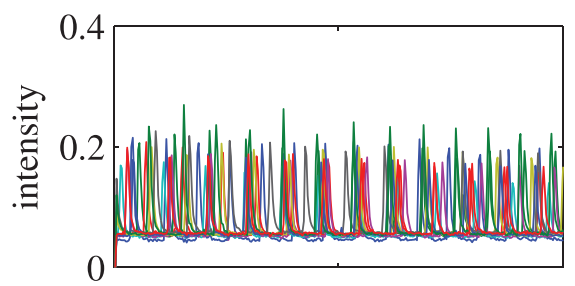

c)

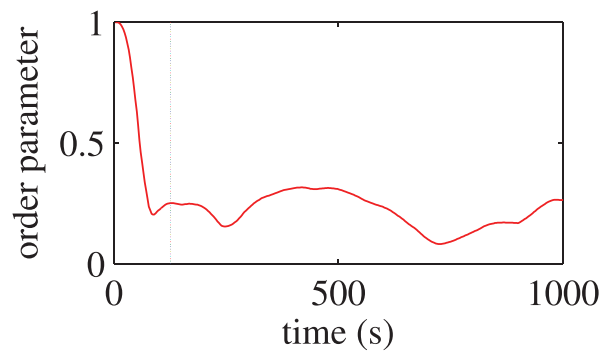

d)

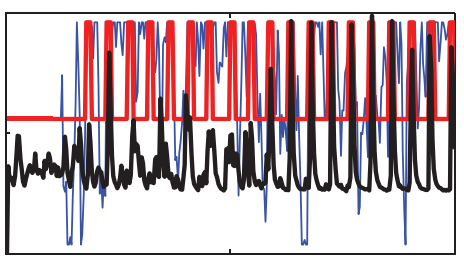

e)

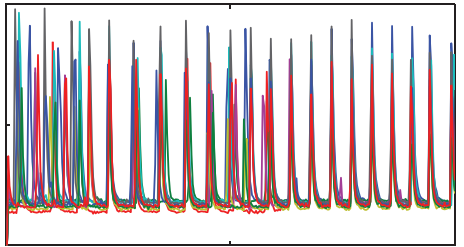

f)

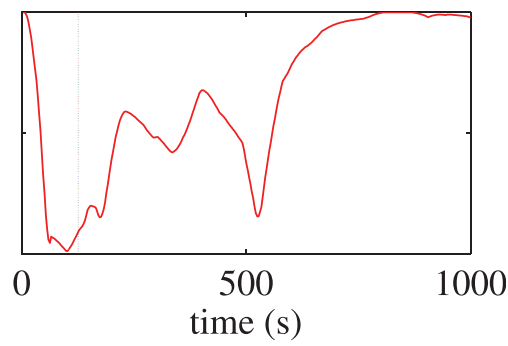

FIG. 5. Effect of periodic perturbations on noise-entrained oscillators. (a) The applied common noise signal, blue line, and the negative pulses, red line. The period of the negative pulse train signal is continually adjusted to be $9 \mathrm{~s}$ shorter than the current period of the control oscillators. The black line is the measured mean signal of all oscillators, excluding the control oscillators. (b) Individual time traces of 10 representative oscillators. (c) Kuramoto order parameter. (d)-(f) Same as (a)-(c) except now using a positive perturbation pulse train. The experiments in (a) and (d) used 30 and 27 oscillators, respectively, and five control oscillators. The negative and positive pulses have a duration of $1 / 4$ of their period. The baseline, positive pulse, and negative pulse light intensities are 140, 240, and 0 gray scale units $(1.29,2.31$, and $0 \mathrm{~mW}$ $\mathrm{cm}^{-2}$ ), respectively. Experimental conditions as in Figure 2. 

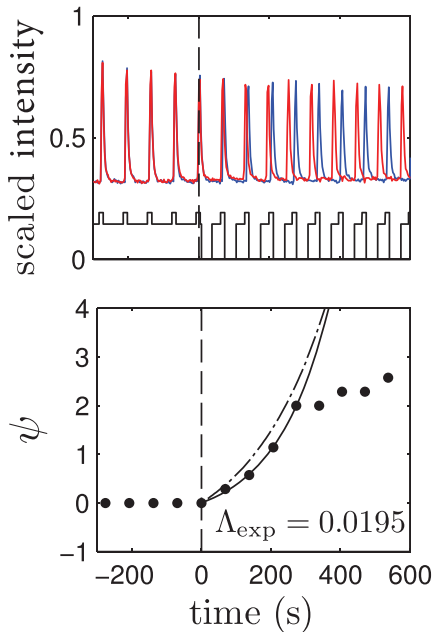
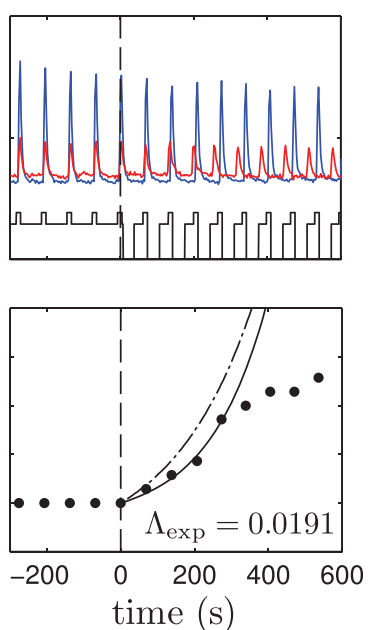
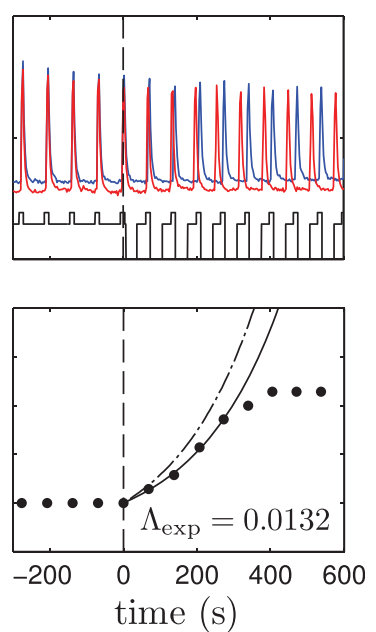

FIG. 6. Analysis of the rate of phase separation. The top panels show traces from individual oscillators as red and blue lines, with the solid black line and dashed line showing the scaled external light intensity and time at which the desynchronizing stimulus is turned on, respectively. Exponential desynchronization is clearly demonstrated in the bottom panels, which show the experimentally observed phase difference, $\psi$, between the oscillators as a function of time, from the top panels. The dotted-dashed line gives the expected desynchronization with values of $\Lambda$ and $\Omega$ calculated from (1), and the solid line is a function of the same form as (1) fitted to the experimental data. Experimental data from Figure 3.

Figure 3. If the desynchronization occurs by inducing chaos, then it is expected that the rate of separation of the oscillations will be exponential in time. The time constant of the separation is determined by the positive Lyapunov exponent. As outlined in Sec. III and based upon the data shown in Figure 3, where the oscillator period is $66 \mathrm{~s}$ and the dark signal is applied for a duration of $33 \mathrm{~s}$, the theoretical values of $\Lambda$ and $\Omega$ can be determined using Eq. (1) to be $\Lambda=0.0135$ and $\Omega=0.0216$.

Figure 6 compares this theoretical prediction with the experimentally observed phase separation of pairs of oscillators. In the upper panels, red and blue traces show recordings from oscillators within the population, the solid black line shows the scaled background light intensity, and the vertical dashed line represents the time at which the desynchronizing stimulus is turned on. The experimentally observed phase difference, $\psi$, between the oscillators is plotted as individual dots in the lower panels. In the lower panels, the dot-dashed line is the maximum expected phase difference predicted by Eq. (1), with $\Delta \omega$ calculated using $\omega_{2}$ from the fastest control oscillator, having a period of $72 \mathrm{~s}$, and the solid line shows the data fitted to an exponential function with the same form as (1). The experimentally observed Lyapunov exponent, $\Lambda_{\text {exp }}$, is slightly higher than the Lyapunov exponent expected from the phase reduction theory but is generally well within the standard error associated with the determination of the theoretical value. We note that in Figure 6, even though $\Lambda<\Lambda_{\text {exp }}$, the theoretical curve is above the experimental curve because the theoretical value of $\Omega$ is larger than what is observed.

\section{DISCUSSION}

In this paper, we demonstrate in an experimental model that phasic stimulation, selected by the PRC, can be used to increase or decrease synchrony in a population of oscillators synchronized by a common periodic or stochastic input. The optimal timing of a desynchronizing signal can be determined directly from the PRC of the dynamical oscillator. In the first set of experiments, using a fully entrained set of oscillators, it is necessary to target the phase region with an associated positive Lyapunov exponent (positive slope in the PRC) to effect phase separation. The desynchronization occurs due to a phase advance of the faster oscillators relative to the slower oscillators, with the slower oscillators remaining entrained to the synchronizing periodic signal.

Stochastic synchronization is thought to generate synchrony in uncoupled oscillators in many natural systems such as brain activity and ecological communities. In this work, we have demonstrated that stochastic synchronization can lead to statistical synchronization in a population of uncoupled experimental oscillators. The intermittent phase alignment occurring through phase resetting by the synchronizing noise leads to a statistically larger average order parameter. In this chemical system, both positive and negative noise perturbations can lead to a degree of phase resetting if they happen to occur towards the end of the phase cycle of the individual oscillators. Theoretical work predicts that stochastic synchronization will be more effective in oscillatory systems with underlying type II PRCs, which take both positive and negative values, compared to systems with type I PRCs, with only positive or only negative values. ${ }^{20,48}$ In our case, the experimental system is a type I PRC but still demonstrates phase alignment through noise.

When applying a negative pulse train to a stochastically synchronized system, there is a distribution of phases of the oscillators at the time of the stimulus. Since the negative perturbation PRC has both a negative and positive region, it will tend to both push and pull the oscillators together and apart. By applying the stimulus at certain phases of the oscillation, the stimulus can counteract the effect of the noise induced synchronization. The resultant order is found to lie intermediate between the stochastic synchronized state and the fully uncoupled incoherent state. Our work demonstrates the resultant state to be significantly different from both. It should be noted, however, that other dynamics may be influencing our chosen order measure, such as transient phase cluster states. ${ }^{25}$ 
In future continuation of this work, it will be of interest to compare the synchronization properties of the experimental stochastically synchronized system with theoretically predicted properties. These properties, which are commonly designated through the probability density function of the phase separation of the oscillators, are expected to depend on characteristics of the frequency distribution of the applied common noise. ${ }^{20}$ Our system could also be used to explore the selection of noise filters for optimal stochastic synchronization and selection of cluster states. A better understanding of the underlying stochastic synchronization will allow for a clearer understanding of how the application of the periodic negative perturbations results in a net desynchronizing effect.

This paper illustrates with a chemical system how stimulus waveforms phase locked to an oscillation can both advance and retard oscillation phase. In the nervous system, increasing synchrony may enhance physiological oscillations and may be used to improve memory, or alternatively, decreasing synchrony may be used to mitigate pathological oscillations seen in Parkinson's disease. Chaotic desychronization achieved through phase response curve selection of the stimulus phases that have the steepest positive slope (for desynchronization) or steepest negative slope (for synchronization) provides a framework for optimizing stimulation parameters and waveforms for closed loop stimulation. This approach may have significant benefits for clinicians in tuning parameters for biomedical devices or in industry where mechanical or chemical oscillations are unwanted.

\section{ACKNOWLEDGMENTS}

This material was based on work supported by the National Science Foundation, Grant Nos. CHE-1212558, CBET-1265435, CBET-1264535, and CBET-0954797.

\section{APPENDIX: SUPPORTING THEORY FOR EXPERIMENTAL VALIDATION OF EXPONENTIAL DESYNCHRONIZATION}

When the desynchronizing stimulus is turned on, we find that the synchronized oscillators split into two populations at an exponential rate. To explain this phenomenon, consider the phase models ${ }^{49}$ of two oscillators

$$
\begin{aligned}
& \dot{\phi}_{1}=\omega_{1}+Z_{-}\left(\phi_{1}\right) u_{-}(t)+Z_{+}\left(\phi_{1}\right) u_{+}(t), \\
& \dot{\phi}_{2}=\omega_{2}+Z_{-}\left(\phi_{2}\right) u_{-}(t)+Z_{+}\left(\phi_{2}\right) u_{+}(t) .
\end{aligned}
$$

Here, $\phi_{1}$ and $\phi_{2}$, both $\in[0,2 \pi)$ give the phase of each respective oscillator, $\omega_{1}$ and $\omega_{2}$ are the natural frequency of each oscillator, and $Z_{-}(\phi)$ and $Z_{+}(\phi)$ are the phase response curves associated with common negative and positive perturbations $u_{-}(t)$ and $u_{+}(t)$, respectively. We note that because $u_{-}(t)$ and $u_{+}(t)$ are both perturbations with respect to the baseline light intensity, it is not possible for both to be nonzero at the same time. In general, the phase response curves for a single oscillator can be measured through the "direct method." 44,45 To obtain a single data point using this strategy, a short pulse of light with duration $\Delta t$ and magnitude $u$ is applied to an oscillator at a random phase $\phi$, the phase change, $\Delta \phi$, is measured by observing the change in spike time, and the resulting data point is $Z(\phi)=\frac{\Delta \phi}{u \Delta t}$. We use this strategy to calculate $Z_{-}(\phi)$, with results shown in Figure 2 from the main text. We can quantify the effect of $Z_{+}(\phi)$ by noting that when a positive pulse is given later in the phase, the resulting change of phase is close to the causality line, ${ }^{50}$ meaning that the positive pulse immediately elicits a spike in the range $\pi \leq \phi \leq 2 \pi$. We also note that $Z_{+}(\phi) \approx 0$ in the range $0 \leq \phi \leq \pi / 2$. To proceed, we define a new variable, $\psi=\phi_{2}-\phi_{1}$ so that

$$
\begin{aligned}
\dot{\psi}= & \Delta \omega+Z_{-}\left(\phi_{2}\right) u_{-}(t)+Z_{+}\left(\phi_{2}\right) u_{+}(t) \\
& -\left(Z_{-}\left(\phi_{1}\right) u_{-}(t)+Z_{+}\left(\phi_{1}\right) u_{+}(t)\right),
\end{aligned}
$$

where $\Delta \omega=\omega_{2}-\omega_{1}$.

In experiments, one group of oscillators remains phase locked to the positive pulses when exponential desynchronization is observed. When the negative, desynchronizing perturbation is turned on, as we see from the PRC in Figure 2, it has a net effect of speeding up the oscillation of each oscillator. When this happens, oscillators with a relatively fast natural frequency will be able to spike (i.e., rapidly increase in amplitude) before the positive perturbation is applied, escaping its synchronizing influence. Conversely, oscillators with a relatively slow natural frequency will not fire before the positive perturbation is applied and will remain synchronized. Without loss of generality, we will take $\phi_{1}$ to be the phase locked oscillator. One can verify that there is no observable difference between the phase locked oscillator and an unforced oscillator with $\omega_{1}=2 \pi / T$, with $T$ being the forcing frequency. We also find that the desynchronizing stimulus speeds up the oscillation of the second oscillator so that when the positive light perturbation is given, $\phi_{2} \geq 0$. If this was not the case, the positive perturbation would cause both oscillators to spike at the same time, maintaining synchronization indefinitely. Averaging theory ${ }^{51,52}$ allows us to approximate $\psi$ by a new variable $\varphi$, where

$$
\begin{aligned}
& \dot{\varphi}=\frac{1}{T} \int_{0}^{T}\left(\Delta \omega+Z_{-}\left(\phi_{2}\right) u_{-}(t)+Z_{+}\left(\phi_{2}\right) u_{+}(t)\right. \\
& \left.-\left(Z_{-}\left(\phi_{1}\right) u_{-}(t)+Z_{+}\left(\phi_{1}\right) u_{+}(t)\right)\right) d t \\
& =\Delta \omega+\frac{1}{T} \int_{0}^{T}\left(Z_{-}\left(\phi_{2}\right) u_{-}(t)\right) d t \\
& \approx \Delta \omega+\frac{1}{T} \int_{0}^{T}\left(Z_{-}\left(\varphi+\phi_{1}\right) u_{-}(t)\right) d t \\
& =\Delta \omega+\underbrace{\frac{1}{T} \int_{0}^{T}\left(Z_{-}\left(\phi_{1}\right) u_{-}(t)\right)}_{\Omega} \\
& +\underbrace{\frac{1}{T} \int_{0}^{T}\left(Z_{-}^{\prime}\left(\phi_{1}\right) u_{-}(t)\right) d t}_{\Lambda} \varphi+\mathcal{O}\left(\varphi^{2}\right) \\
& =\Delta \omega+\Omega+\Lambda \varphi+\mathcal{O}\left(\varphi^{2}\right) \text {. }
\end{aligned}
$$

Note that in the second line, the dynamics of the phase locked oscillator $\phi_{1}$ is approximated by an unforced oscillator, as mentioned earlier. We have also used the fact that 
$Z_{+}\left(\phi_{2}\right) \approx 0$ when the positive pulse is applied. In the fourth line, we have used the Taylor expansion about $Z_{-}\left(\phi_{1}\right)$ so that ${ }^{\prime} \equiv d / d \phi$. Here, $\Lambda$ is the finite time Lyapunov exponent (cf. Ref. 43) which determines the rate of exponential desynchronization. Note that the equation for $\varphi$ approximates $\psi$ best when the external inputs are small. Finally, to leading order in $\varphi$, the solution to Equation (A3) is

$$
\varphi(t)=\frac{\Delta \omega+\Omega}{\Lambda}\left(e^{\Lambda t}-1\right) \approx \psi(t) .
$$

Above, we have assumed $t=0$ corresponds to the instant that the oscillators spike together right before the desynchronizing stimulus is applied so that $\varphi(0)=0$. The values $\Lambda$ and $\Omega$ can be approximated using $\phi_{1}(t)=\omega_{1} t$, as was used in Ref. 28, which is valid in the limit that the magnitude of the external stimuli is small. We note that the preceding derivation is unique to the $\mathrm{BZ}$ system; a more general discussion of desynchronization using finite time Lyapunov exponents can be found in Refs. 28 and 29.

${ }^{1}$ C. M. Gray, P. König, A. K. Engel, and W. Singer, Nature 338, 334 (1989).

${ }^{2}$ G. Copinschi, K. Spiegel, R. Leproult, and E. Van Cauter, Novartis Found. Symp. 227, 143 (2000).

${ }^{3}$ J. O'Keefe and M. L. Recce, Hippocampus 3, 317 (1993).

${ }^{4}$ J. Beck-Friis, D. von Rosen, B. F. Kjellman, J. G. Ljunggren, and L. Wetterberg, Psychoneuroendocrinology 9, 261 (1984).

${ }^{5}$ P. Brown, Curr. Opin. Neurobiol. 17, 656 (2007).

${ }^{6}$ J. Raethjen, R. B. Govindan, F. Kopper, M. Muthuraman, and G. Deuschl, J. Neurophysiol. 97, 3219 (2007).

${ }^{7}$ A. J. Lewy, R. L. Sack, and C. M. Singer, Ciba Found. Symp. 117, 231 (1985).

${ }^{8}$ A. A. Kühn, F. Kempf, C. Brücke, L. Gaynor Doyle, I. Martinez-Torres, A. Pogosyan, T. Trottenberg, A. Kupsch, G. H. Schneider, M. I. Hariz et al., J. Neurosci. 28, 6165 (2008).

${ }^{9}$ W. C. Koller, P. R. Pahwa, K. E. Lyons, and S. B. Wilkinson, Neurology 55, S29 (2000).

${ }^{10}$ T. A. Pedley and R. D. Traub, Physiological Basis of EEG (Raven Press, New York, 1990), vol. 2, p. 824.

${ }^{11}$ C. Hammond, H. Bergman, and P. Brown, Trends Neurosci. 30, 357 (2007).

${ }^{12}$ A. Pikovsky, M. Rosenblum, and J. Kurths, Synchronization: A Universal Concept in Nonlinear Sciences (Cambridge University Press, 2003), vol. 45 .

${ }^{13}$ H. Nakao, K. Arai, and Y. Kawamura, Phys. Rev. Lett. 98, 184101 (2007).

${ }^{14}$ D. S. Goldobin, J. N. Teramae, H. Nakao, and G. B. Ermentrout, Phys. Rev. Lett. 105, 154101 (2010).

${ }^{15}$ J.-N. Teramae and D. Tanaka, Prog. Theor. Phys. Supp. 161, 360 (2006).

${ }^{16}$ C. Zhou, J. Kurths, I. Z. Kiss, and J. L. Hudson, Phys. Rev. Lett. 89, 014101 (2002).

${ }^{17}$ R. F. Galán, G. B. Ermentrout, and N. N. Urban, Sens. Actuators, B 116, 168 (2006).

${ }^{18}$ P. Tass, J. Biol. Phys. 22, 27 (1996).
${ }^{19}$ W. Kurebayashi, K. Fujiwara, and T. Ikeguchi, Europhys. Lett. 97, 50009 (2012).

${ }^{20}$ R. F. Galán, G. B. Ermentrout, and N. N. Urban, Phys. Rev. E 76, 056110 (2007).

${ }^{21}$ S. Hata, K. Arai, R. F. Galán, and H. Nakao, Phys. Rev. E 84, 016229 (2011).

${ }^{22}$ R. F. Galán, N. Fourcaud-Trocmé, G. B. Ermentrout, and N. N. Urban, J. Neurosci. 26, 3646 (2006).

${ }^{23}$ A. B. Neiman and D. F. Russell, Phys. Rev. Lett. 88, 138103 (2002).

${ }^{24}$ Y. L. Maistrenko, O. V. Popovych, and P. A. Tass, Desynchronization and Chaos in the Kuramoto Model, Lecture Notes in Physics vol. 671 (Springer, 2005), pp. 285-306.

${ }^{25}$ P. Tass, Europhys. Lett. 59, 199 (2002).

${ }^{26}$ Y. Zhai, I. Z. Kiss, P. A. Tass, and J. L. Hudson, Phys. Rev. E 71, 065202 (2005).

${ }^{27}$ P. Tass, Biol. Cybernet. 85, 343 (2001).

${ }^{28}$ D. Wilson and J. Moehlis, J. Comput. Neurosci. 37, 243 (2014).

${ }^{29}$ D. Wilson and J. Moehlis, SIAM J. Appl. Dyn. Syst. 13, 276 (2014).

${ }^{30}$ O. V. Popovych, C. Hauptmann, and P. Tass, Phys. Rev. Lett. 94, 164102 (2005).

${ }^{31}$ Y. Zhai, I. Z. Kiss, H. Kori, and J. L. Hudson, Physica D 239, 848 (2010).

${ }^{32}$ C. J. Wilson, B. Beverlin, and T. Netoff, Fron. Syst. Neurosci. 5, 50 (2011).

${ }^{33}$ A. Holt and T. Netoff, BMC Neurosci. 14, 291 (2013).

${ }^{34}$ A. F. Taylor, P. Kapetanopoulos, B. J. Whitaker, R. Toth, L. Bull, and M. R. Tinsley, Euro. Phys. J. Spec. Top. 165, 137 (2008).

${ }^{35}$ R. J. Field, E. Körös, and R. M. Noyes, J. Am. Chem. Soc. 94, 8649 (1972).

${ }^{36}$ A. F. Taylor, Prog. Reac. Kinet. Mech. 27, 247 (2002).

${ }^{37}$ A. F. Taylor, M. R. Tinsley, and K. Showalter, Phys. Chem. Chem. Phys. 17, 20047 (2015).

${ }^{38}$ K. Showalter and I. R. Epstein, Chaos 25, 097613 (2015).

${ }^{39}$ J. Maselko, J. Reckley, and K. Showalter, J. Phys. Chem. 93, 2774 (1989).

${ }^{40}$ R. Toth and A. F. Taylor, Prog. Reac. Kinet. Mech. 31, 59 (2006).

${ }^{41}$ M. R. Tinsley, S. Nkomo, and K. Showalter, Nat. Phys. 8, 662 (2012).

${ }^{42}$ S. Nkomo, M. R. Tinsley, and K. Showalter, Phys. Rev. Lett. 110, 244102 (2013).

${ }^{43}$ D. Wilson and J. Moehlis, Biophys. J. 107, 1744 (2014).

${ }^{44}$ E. M. Izhikevich, Dynamical Systems in Neuroscience: The Geometry of Excitability and Bursting (MIT Press, London, 2007).

${ }^{45}$ T. Netoff, M. Schwemmer, and T. Lewis, in Phase Response Curves in Neuroscience, edited by N. Schultheiss, A. Prinz, and R. Butera (Springer, New York, 2012), pp. 95-129.

${ }^{46}$ K. Yoshimura, I. Valiusaityte, and P. Davis, Phys. Rev. E 75, 026208 (2007).

${ }^{47}$ D. Gillespie, Phys. Rev. E 54, 2084 (1996).

${ }^{48}$ A. Abouzeid and B. Ermentrout, Phys. Rev. E 80, 011911 (2009).

${ }^{49}$ F. C. Hoppensteadt and E. M. Izhikevich, Weakly Connected Neural Networks (Springer, 1997).

${ }^{50}$ A. Nabi, T. Stigen, J. Moehlis, and T. Netoff, J. Neural Eng. 10, 36005 (2013).

${ }^{51}$ J. A. Sanders, F. Verhulst, and J. Murdock, Averaging Methods in Nonlinear Dynamical Systems, 2nd ed. (Springer-Verlag, New York, 2007).

${ }^{52}$ J. Guckenheimer and P. Holmes, Nonlinear Oscillations, Dynamical Systems, and Bifurcations of Vector Fields (Springer Verlag, New York, 1983), vol. 42. 